\title{
Psychological and Social Effects of Orthodontic Treatment
}

\author{
Judith E. N. Albino, ${ }^{1,4}$ Sandra D. Lawrence, ${ }^{2}$ \\ and Lisa A. Tedesco ${ }^{3}$
}

Accepted for publication: April 7, 1993

Adolescents with commonly occurring forms of malocclusion often are presumed to be at risk for negative self-esteem and social maladjustment. $A$ randomized control group design was used to assess the psychosocial effects of orthodontic treatment for esthetic impairment. Ninety-three participants, 11 to 14 years old, with mild to moderate malocclusions, were randomly assigned to receive orthodontic treatment immediately or after serving as delayed controls. $A$ battery of psychological and social measures was administered before treatment, during treatment, and three times after completion of treatment, the last occurring one year after termination. Repeated measures analyses of variance assessed group differences at the five time points. Parent-, peer-, and self-evaluations of dental-facial attractiveness significantly improved after treatment, but treatment did not affect parent- and self-reported social competency or social goals, nor subjects' self-esteem. In summary, dental-specific evaluations appear to be influenced by treatment, while more general psychosocial responses are not.

KEY WORDS: orthodontic treatment; malocclusions; adolescence; psychosocial effects.

This research was supported by Grant NIH-NIDR-R01-DE06154 from the National Institute of Dental Research.

'Departments of Applied Dentistry and Psychology, University of Colorado, Boulder, Colorado 80309.

${ }^{2}$ Psychological Service at National Rehabilitation Hospital, Washington, DC.

${ }^{3}$ Department of Periodontics, Prevention, and Geriatrics, University of Michigan, Ann Arbor, Michigan 48109.

${ }^{4}$ To whom correspondence should be addressed at Office of the President, University of Colorado, 914 Broadway, Campus Box 35, Boulder, Colorado 80309-0035. 


\section{INTRODUCTION}

Malocclusion, the broad range of frequently occurring dental-facial malrelations, refers to physical deviations from ideal occlusal relations and functioning. Yet the effects experienced by those suffering from malocclusion frequently are described entirely in terms of psychosocial concerns (Fisk, 1963; Macgregor, 1970; Pitt, 1977; Secord and Backman, 1959; Shaw, 1981; Shaw et al., 1980a, b; Stricker, 1970; Stricker et al., 1979). Indeed, there is now general agreement within the orthodontic profession that a clinical determination of "need for treatment" should include consideration of psychosocial, as well as physical, effects of malocclusion. However, neither psychosocial effects of dental-facial malrelations nor psychosocial responses to orthodontic treatment have been demonstrated in systematic research. The present study answers basic questions about the effects of orthodontic treatment and provides information important to health policy decisions.

In recent years, the use of public funds to provide orthodontic treatment has emphasized the need to verify the deleterious psychological and social effects of malocclusion and determine the degree to which they are alleviated by orthodontic treatment. Estimates based on epidemiological research indicate that at least $70 \%$ of the population is affected by some form of occlusal malrelations (King, 1983; McLain and Proffitt, 1985). Moreover, surveys indicate that nearly $50 \%$ of U.S. children would benefit from treatment and that some 5\% of these are seriously handicapped (Kelly and Harvey, 1977). It should be noted that among those who actually seek treatment, an estimated $80 \%$ do so for cosmetic reasons (Rosenberg, 1974).

Research involving procedures for evaluating dental-facial attractiveness has shown that when other facial features are held constant, normal occlusion is perceived as more attractive than various forms of malocclusion. In most of these studies the effects of malocclusion were depicted in drawings or photographs showing various types of occlusion (Albino, 1981; Cohen and Horowitz, 1970; Jenny et al., 1980; Lucker et al., 1981; Prahl-Andersen, 1978; Shaw, 1981; Tedesco et al., 1983a, b). Gochman $(1972,1975)$ also used drawings in his studies and showed that most children prefer straight, evenly spaced teeth with noticeably carious lesions to healthy, but crowded and poorly aligned dentition.

Shaw et al. (1980b) found that teeth represented the fourth most common target of teasing for children 9 to 12 , after height, weight, and hair. Interestingly, teasing about the teeth resulted in strong feelings of upset and the sense of being harassed more often than did other types of teasing. Shaw (1981) observed that both children and adults identified faces with normal occlusion as more attractive, more intelligent, less inclined to aggression, and more desirable as friends than identical faces with occlusal impairment. 
In other studies, investigators have attempted to demonstrate the effects of malocclusion on psychosocial functioning by examining changes in self-image and other personality measures following orthodontic treatment. In comparing persons 5 years after completion of orthodontic treatment with individuals who had not received treatment for diagnosed malocclusions, Rutzen (1973) discovered that subjects did not differ on several personality measures. Although those treated reported significantly more positive assessments of their appearance and had lower levels of anxiety, they did not obtain higher scores on Rosenberg's Self-Esteem Scale or on measures of extraversion or neuroticism. Unfortunately, Rutzen's work did not include pretreatment comparisons and it is impossible to determine whether the effects identified are due to treatment or merely to sample differences.

Dennington and Korabik (1977) found positive changes on the Tennessee Self-Concept Scale for patients measured before treatment and 7 months after banding. They had no controls, however, and no posttreatment data. Klima et al. (1979), on the other hand, found no significant differences among orthodontic patients in retention, prospective patients, and nonpatients on measures of body image or self-concept. Their study, however, did not control for objectively evaluated dental-facial appearance or for other potential mediating variables.

Research demonstrating the positive relationship between physical attractiveness and interpersonal popularity, as well as others' favorable evaluations of personality, social behaviors, and intellectual expression strongly suggests that malocclusion may have important social consequences and psychological effects (Barocas and Daroly, 1972; Berscheid and Walster, 1974; Berscheid et al., 1971; Dion et al., 1972; Goldman and Lewis, 1977). MacGregor (1970) suggested that children with these milder forms of facial disfigurement may actually be at greater risk for developing psychological problems. In such cases, the inconsistency of others' responses to their appearance creates high levels of uncertainty that may result in anxiety. Yet data directly addressing the psychosocial responses to malocclusion are sparse.

In summary, normal occlusion generally is perceived as more attractive than dental-facial malrelations, both by those affected and by others. In addition, it appears that there is at least some negative social feedback associated with highly visible and less attractive forms of malocclusion. However, because malocclusions often are highly visible and yet may not represent an overriding determinant of facial attractiveness, responses may be difficult to assess. Data indicate that responses either by the individual affected or by those with whom he or she associates probably will not be strongly related to the objective or clinical severity of occlusal impairment. 
It appears that malocclusion must be assessed in terms of selfperceptions as well as objective evaluations of appearance and that responses to dental-facial characteristics, rather than total appearance, are most relevant. The poor contribution of personality measures in previous studies suggests the need to focus more on specific personal attitudes and behaviors that may be influenced by changes in dentalfacial appearance.

Within the context of a broader study of psychosocial factors in malocclusion (Albino, 1990), we asked whether or not adolescents who undergo orthodontic treatment experience positive changes in psychosocial functioning. The hypotheses below were tested using a randomized control group design.

When compared to those who do not receive orthodontic treatment, adolescents who receive treatment will

(1) receive more positive evaluations of dental-facial attractiveness after treatment completion;

(2) demonstrate more positive perceptions of their occlusal appearance;

(3) report more frequent self-initiated social involvement with peers;

(4) demonstrate more appropriate social behavior, as reported by parents;

(5) demonstrate higher self-esteem.

\section{METHOD}

This study utilized an experimental design in which 93 adolescents with diagnosed malocclusions were randomly assigned to Treatment and Control Groups. The Treatment Group received immediate orthodontic treatment to correct their occlusal malrelations, while treatment for the Control Group was delayed until after final data collection.

The effects of treatment on dental-facial appearance and on psychosocial functioning in study participants were evaluated by comparing scores for the two groups on a battery of psychosocial measures. These measures were obtained: (1) before Treatment Group subjects began orthodontic treatment; (2) 8 to 10 months after this treatment began but, in all cases, before treatment ended; (3) on termination of active treatment (removal of fixed appliances); (4) 6 months after termination; and (5) 1 year after termination of treatment. Control Group subjects were randomly assigned to a comparable schedule. Data from one instrument (Body Image) were not collected prior to treatment because they were not incorporated into the study until after treatment had begun. 


\section{Subjects}

Study participants were 46 girls and 47 boys between 11 and 14 years of age when the study began (mean age, 12.5 years). All had been evaluated for orthodontic treatment need at either the Erie County (New York) Orthodontic Screening Clinic or the State University of New York at Buffalo Orthodontic Screening Clinic.

Adolescents who had applied at the County Clinic and were denied treatment were invited to participate in this study. Even though they did not have orthodontic conditions disabling enough to warrant financial assistance, they were judged as having mild to moderate malocclusions that would benefit from treatment. Subjects recruited at the University Clinic met similar treatment criteria but did not meet the specific teaching needs of orthodontic residents at this clinic. Potential study subjects were offered free treatment with the understanding that they would be randomly selected for either the immediate- or the delayed-treatment group. All had been diagnosed with mild to moderate malocclusion, and in every case the judgment was made that delayed treatment would not compromise the long-term prognosis. Control Group subjects were monitored for detrimental changes in occlusion by orthodontists throughout the waiting period.

Those evaluated at the County Clinic were referred by school dental health personnel or by dentists in private practice who had identified potential orthodontic problems. The Erie County screening clinic was responsible for a large and well-known program through which all cases were assigned to private orthodontists, with support provided on a sliding scale. Thus, the range of incomes and health care experience of the patients reflected a broader sampling than would be expected in most public assistance populations. Applicants applying to the University Orthodontic Clinic comprised a similarly broad-based and representative group, including potential patients attracted by the clinic's reputation for excellence in orthodontic care, as well as the somewhat lower fee schedule than is available in the private sector.

At the time of the initial assessment in 1984, subjects' family incomes ranged from "less than $\$ 10,000$ " to "more than $\$ 40,000$," as determined by self-identified income ranges. Since no upper limit was set, a true median cannot be determined. Using this method, however, the median yearly income range for all participants was estimated to be in the $\$ 15$ to $\$ 20,000$ range, which was similar to relevant local indices at the time of the study. Males and females participated, and 10 of the 93 subjects did not describe themselves as Caucasian. 
Of the 93 subjects who began, 76 completed the study, 39 of 44 in the Treatment Group and 37 of 49 in the Control Group. Ten Control Group subjects decided to seek treatment elsewhere because of the delay related to their group assignment. Other reasons for attrition included moves from the area, personal considerations (e.g., family problems), and in two cases, repeated failures of the subjects to keep appointments for follow-up assessments.

\section{Procedure}

Prior to beginning treatment, each subject and one parent individually completed a structured interview that included a battery of psychosocial and orthodontic-specific measures. Subjects were then randomly assigned to the Treatment or Control Group and given a brief orthodontic examination to determine the degree of malocclusion.

All subjects in the Treatment Group were interviewed 8 to 10 months after beginning treatment to assess effects related to wearing orthodontic appliances. Following completion of active treatment (removal of fixed appliances), data were collected at three intervals (immediately, after 6 months, and after 1 year), allowing assessment of both short-term and more enduring responses to treatment. Posttreatment interviews were individually scheduled for all subjects. This form of scheduling was deliberately chosen in an effort to best address the normal variations in duration of orthodontic treatment. Each time a Treatment Group subject reached the first posttreatment assessment, a Control Group subject was randomly selected for parallel assessment. These pairings were maintained for later assessments as well. After the 1-year follow-up data had been collected for a given Control Group subject, that individual was scheduled to begin orthodontic treatment. Further data were not collected for Control Group subjects.

Treatment for all participants was provided by the Faculty Practice Group of the SUNY-Buffalo Orthodontics Department, using a standard method relying on straight wire modifications of edgewise mechanotherapy. These procedures included banding or bonding all patients' teeth and any arch wires that were needed. Adjunctive mechanical requirements such as head gear, holding arches, lip bumpers, or sutural expansion appliances were used as dictated by individual patient requirements.

\section{Description of Measures}

To evaluate the five earlier-stated hypotheses describing the relationship between psychosocial variables and orthodontic treatment, instruments 
were selected to assess self-esteem and self-concept, as well as reports of social behaviors and goals. Body image and perceptions of facial attractiveness also were assessed. Both study participants and their parents were asked to respond. Most of these instruments were developed by the authors specifically for use with adolescents seeking or involved in orthodontic treatment. Data on their psychometric properties, therefore, are available in the cited literature, which also provides descriptions of the development of the tests and facilitates full understanding of the constructs being measured. Internal reliabilities calculated for the current sample met criteria established in the test development process - generally $\alpha \geq 80$. Further information about the interrelationships of these variables, as well as other measurement characteristics, is given by Albino and Tedesco (1988).

In addition to measures of dependent variables, the Crandall Social Desirability Scale (Crandall et al., 1965) was used as a covariate to control for participants' needs to conform to social values and expectations. The Treatment Priority Index (Grainger, 1967), a measure of clinical malocclusion, was used as a baseline to confirm comparability of groups on severity of orthodontic condition.

\section{Psychosocial - Child}

Coopersmith Self-Esteem Inventory. This widely used, self-administered 10-item measure assesses the adolescent's sense of personal self-worth and competence in relationships. Psychometric data are discussed by Coopersmith (1984). A high score indicates positive self-esteem.

Rosenberg Self-Image Inventory. This 38-item interviewer-administered inventory surveys adolescent behaviors reflecting self-concept along psychological, social, and physical dimensions. This scale has been widely used and tested across age groups and its psychometric properties are reviewed by Simmons et al. (1973). Subscales include Self-Consciousness, Stability of Self-Evaluations, Self-Esteem, Content of Self-Image, Perceived SelfImage, and Depressive Affect. A high score indicates a higher degree of the attribute being measured.

Social Competence and Goals. This self-administered, 40-item, 5point Likert-type questionnaire measures the extent to which the subject perceives him-/herself as comfortable in social environments and the extent to which he/she chooses social versus nonsocial goals (Ford, 1982). A high score indicates that the child chooses social goals and is more socially competent. 


\section{Orthodontic Specific - Child}

Body-Image. This self-administered instrument measures the child's satisfaction with and perceived importance of 27 aspects of appearance, including the chin and teeth. It includes Kiyak and co-workers' (1982) modification of Secord and Jourard's (1953) body cathexis scale. A high score indicates that the child attributes greater satisfaction and importance to the body part being measured. This measure provided an assessment of perceived dental-facial appearance within the context of self-evaluations of other aspects of physical appearance. For the current investigation, items focusing on the teeth and chin were utilized.

Child Perception of Occlusion. This interviewer-administered picture ranking instrument allows the investigators to assess subjects' perceptions of the degree their own occlusion deviates from occlusal appearance judged as most attractive. Psychometric data are discussed by Albino et al. (1979) and Lewis et al. (1979). A high score indicates that the child perceives his/her face as unattractive.

\section{Psychosocial - Parent}

Parent Social Competence and Goals. This self-administered, 40-item, 5 -point Likert-type questionnaire measures the extent to which the parent perceives his/her child as comfortable in social environments and the extent to which the child chooses social versus nonsocial goals (Ford, 1982). A high score indicates that the parent perceives the child as choosing social goals and being more socially competent.

\section{Orthodontic Specific - Parent}

Parent Perception of Occlusion. This interviewer-administered picture ranking instrument assesses parents' perceptions of the degree to which their child's occlusion deviates from occlusal appearance judged as most attractive. Psychometric data are discussed by Albino et al. (1979) and Lewis et al. (1979). A low score indicates greater perceived attractiveness.

\section{Orthodontic Specific - Peers}

Dental-Facial Attractiveness. This interviewer-administered picture rating instrument has independent peer judges rate the relative attractiveness of adolescent participants' occlusal status based on three-quarter-view 
photographs of the participants' smiles. Descriptions of the rating method and data on the reliability and validity of this measure are given by Tedesco et al. $(1983 \mathrm{a}, \mathrm{b})$. Scores on a 5-point scale are obtained using a sorting technique guided by pictures representing each scale point and controlling for race and sex of the rater and participant. Lower scores indicate greater perceived attractiveness.

\section{Psychosocial Covariate - Child}

Crandall Social Desirability Scale. This widely used self-administered 30 -item true-false inventory assesses a child's need for social approval. Psychometric data are discussed by Crandall et al. (1965). A high score indicates a greater need for social approval.

\section{Orthodontic Specific - Orthodontists}

Treatment Priority Index. Developed by Grainger (1967) based on a large national sample of school-age children, this scale was used to assess the severity of malocclusion and to confirm effective randomization for severity of clinical condition. Slakter et al. (1980) have described the use of this instrument with adolescents. A high score indicates a greater degree of malocclusion.

\section{RESULTS}

Initial data were evaluated for group differences using multivariate tests. No significant mean differences between the Treatment Group and the Control Group were found on any of the baseline variables, including baseline malocclusion scores on the Treatment Priority Index. These results confirm that randomization had been achieved by group assignment. No significant gender differences were found in any of the analyses. Effects of race were not assessed because only about $10 \%$ of the participants described themselves as nonCaucasian. Treatment Group and Control Group means and standard deviations for all variables at three data collection points are presented in Table I.

Multivariate repeated-measures analyses of variance were conducted to evaluate between-group differences. $t$ tests were employed for post hoc univariate analyses to tease out components of the interactions that were already identified as significant by the multivariate repeated measures analyses. 


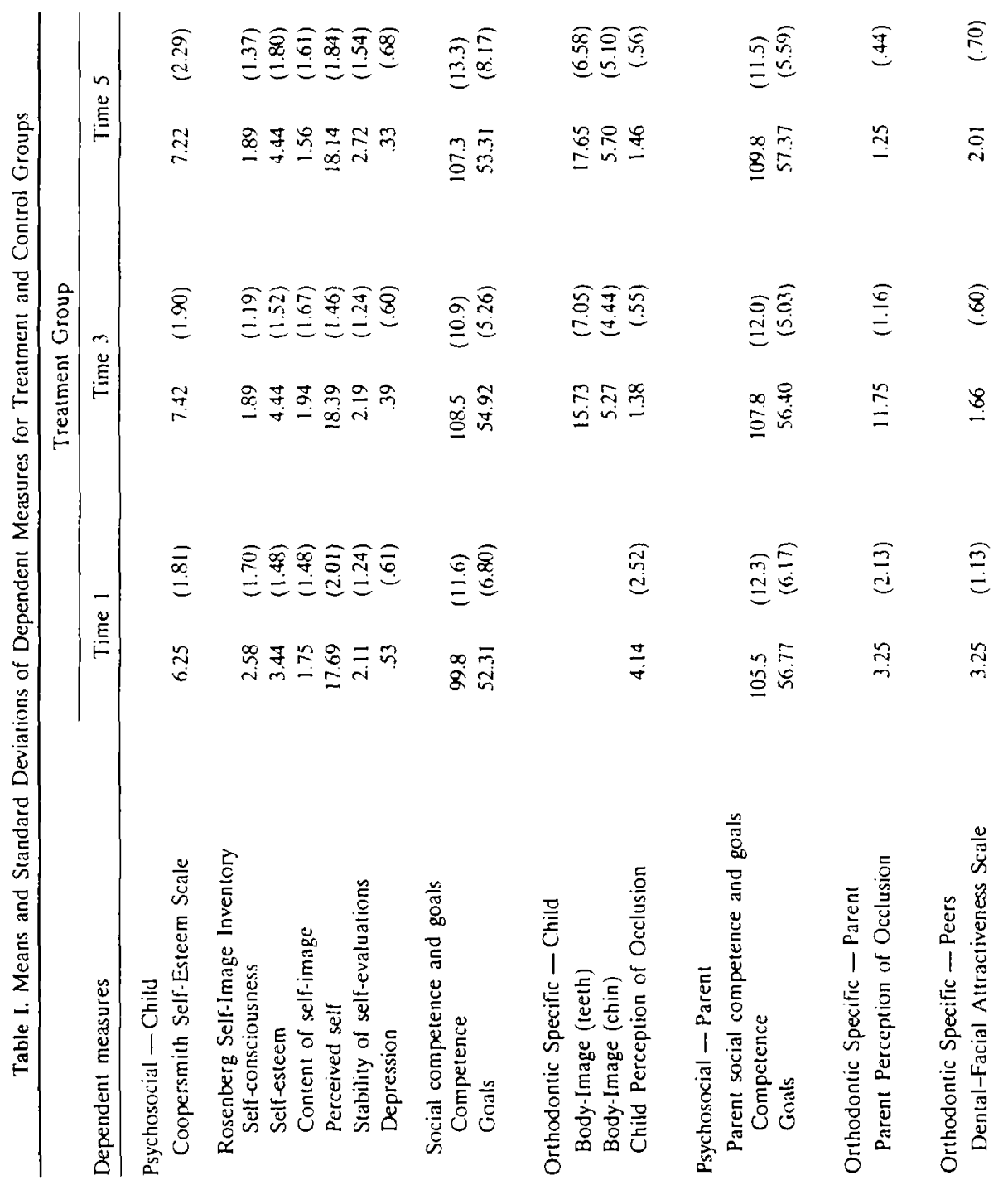




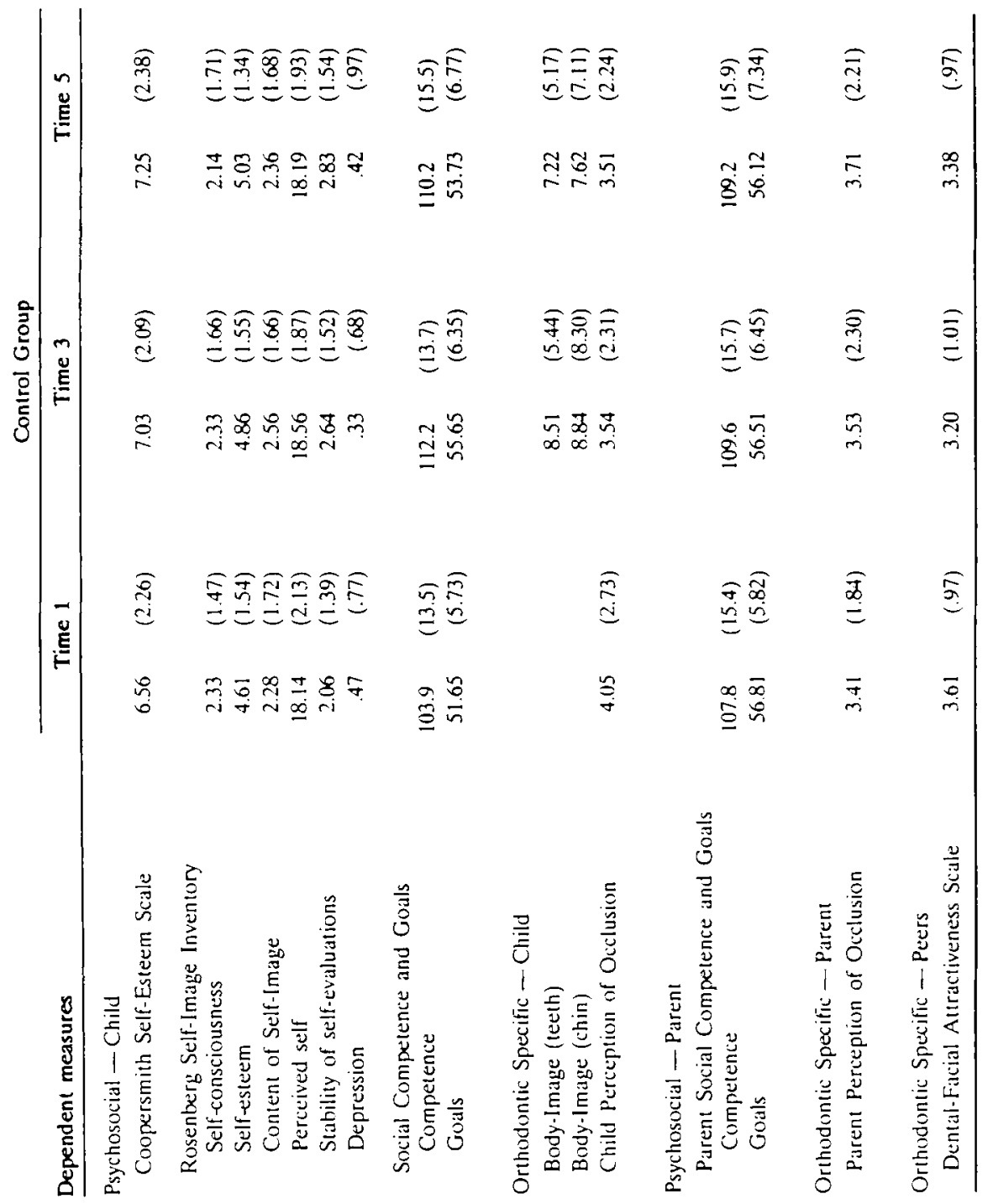


Results of the analyses confirmed the first two hypotheses, but not the other three, as described below.

Hypothesis 1. Compared to those who do not receive orthodontic treatment, adolescents who complete treatment will receive more positive evaluations of dental-facial attractiveness.

Results of a repeated-measures multivariate analysis of variance for Parent Perception of Occlusion (PPO) and Dental-Facial Attractiveness (DFA) indicate a significant group $\times$ time interaction $(F=9.45$, df $=4,61$, $p<.001)$. In addition, the peer evaluations of subjects before treatment were significantly, but not solely, related to the parent perceptions of attractiveness $(r=.39, p<.001)$.

Closer analyses reveal that peer and parent attractiveness ratings did not change significantly over time for the Control Group but improved significantly (lower DFA and PPO scores indicate greater attractiveness) from pre- to immediate posttreatment for the Treatment Group [DFA, $t(31)=$ 7.24, $p<.001$; PPO, $t(31)=3.71, p<.001]$. These effects were maintained 1 year after treatment completion. In other words, parents and peers evaluated the dental-facial attractiveness of Treatment Group participants more positively than that of Control Group participants and these results were maintained over time.

Hypothesis 2. Compared to those who do not receive orthodontic treatment, adolescents who complete treatment will demonstrate more positive perceptions of their occlusal appearance.

Results of repeated measures analysis of variance for Child Perception of Occlusion (CPO) indicate a significant group $\times$ time interaction $(F=7.80, \mathrm{df}=2,71, p<.001)$. Closer analyses reveal that participants' self-evaluations of dental-facial attractiveness did not change significantly over time for the Control Group but improved significantly (lower CPO scores indicate more positive attractiveness) from pre- to immediately posttreatment for the Treatment Group $[t(36)=7.00, p<.001]$. This effect was maintained 1 year after treatment completion $[t(36)=6.36$, $p<.001]$.

A repeated-measures multivariate analysis of variance compared posttreatment self-evaluations of body image related to the teeth and chin and found a significant group effect $(F=39.04, \mathrm{df}=2,71, p<.001)$. The group difference for Body Image (teeth) is consistent with the Child Perception of Occlusion results from the previous analyses. Therefore, posttreatment comparisons indicate that Treatment Group participants evaluated their satisfaction with the importance of their teeth more positively than did those participants for whom treatment was delayed $[t(68)=4.93, p<.001]$. 
The reverse was true, however, for the Body Image (chin) evaluation. Here, posttreatment comparisons indicate that Treatment Group subjects evaluated their satisfaction with and the importance of their chins significantly more negatively than did those participants who had not received treatment $[t(55)=2.31, p<.05]$.

Hypothesis 3. Compared to those who do not receive orthodontic treatment, adolescents who complete treatment will report more frequent self-initiated social involvement with peers.

Since scores on the social competency and social goals measures were highly correlated with social desirability $(r=.39, p<.001)$, scores on the Crandall Social Desirability Scale were covaried to control for participants' needs to conform to social values and expectations. Results of a repeated-measures multivariate analysis of variance indicate a significant effect of time $(F=6.90$, df $=8,64, p<.001)$ on self-reported social goals and social competency. There were, however, no effects of group. Therefore, while the frequency of self-reported social goals and social competency changed over time, these changes cannot be attributed to treatment effects.

Hypothesis 4. Compared to those who do not receive orthodontic treatment, adolescents who complete treatment will demonstrate more social behavior, as reported by parents.

Results of a repeated-measures multivariate analysis of variance indicate no significant effects of time or group on parents' reports of the child's social competency and social goals. This absence of an effect is in contrast to participants' self-reports.

Hypothesis 5. Compared to those who do not receive orthodontic treatment, adolescents who complete treatment will demonstrate higher self-esteem.

Results of a repeated-measures multivariate analysis of variance indicate a significant effect of time $(F=2.18, \mathrm{df}=28,43, p<.01)$ but no effect of group on participants' self-concept. Therefore, while participants' scores improved over time, these changes are not attributable to treatment effects.

\section{DISCUSSION}

Results suggest that orthodontic treatment effectively improves adolescents' own evaluations of their dental-facial attractiveness but that these evaluations do not necessarily generalize to other facial characteristics or to general perception of the face. In fact, participants' evaluation of specific facial characteristics may even worsen immediately 
following treatment, since adolescents who receive orthodontics evaluated their chins more negatively than those who did not. This result may reflect a refocusing of subjects' negative judgments of the appearance of their teeth, which had been fixed, to other aspects of their face (e.g., chin). Improvement in participants' occlusal relations may have resulted in other perceived flaws becoming more salient. These results are consistent with Kiyak and co-workers' (1984) report on psychosocial changes resulting from orthognathic surgery. Parent- and peer evaluations of participants' dental-facial attractiveness also were significantly more positive following treatment. This result suggests that both subjective and relatively objective perceptions of dental-facial attractiveness were positively changed by treatment.

Orthodontic treatment does not appear to improve or change selfand parent evaluations of social competency and social goals. Both the treatment and the delayed groups, however, demonstrated significant changes in these variables over time, an effect that is likely attributable to maturation. These findings are not surprising, since as teenagers mature from early to middle adolescence, they become more autonomous and are therefore able to engage in more peer activities, raise their goals, and become more competent with experience. Middle adolescence represents a time when social relationships may be particularly important - increasing from early adolescence and leveling off in late adolescence (Coleman, 1980). Differences were found in favor of the treatment group for perceptions (e.g., cognitions) but not for behaviors. Clearly, this suggests that orthodontic treatment influences cognitions more readily than behaviors, a finding that mirrors the maturational patterns of adolescence.

Similarly, orthodontic treatment does not seem to affect self-concept in adolescents. It is possible, as Kiyak et al. (1984) suggest, that just knowing one is going to receive treatment boosts self-esteem. Or perhaps a 1-year posttreatment follow-up does not adequately assess psychosocial effects of treatment. Although it is possible that the repeated testing required by the longitudinal design of this study could affect responses to psychosocial measures, the relatively lengthy intervals of 6 months or more between data collection efforts should minimize the potential threat to validity.

The results might lead one to speculate that these adolescents' selfconcepts were not initially affected by their dental-facial condition. A mild to moderate degree of malocclusion may, therefore, result in a limited impact of treatment on self-esteem. In support of this explanation, baseline scores on the subscales of the Rosenberg Self-Image Inventory were comparable to normative data for young adolescents presented by Simmons 
et al. (1973). In addition, the increases in self-esteem and stability of selfimage in both groups from pre- to posttreatment are consistent with Simmons and co-workers' (1973) findings showing that self-image scores generally decreased from early to middle adolescence, before increasing to their previously higher levels later in the teenage years.

It is difficult to believe that documented changes in self-perceived dental-facial attractiveness have no effect on self-concept, especially in a culture that places so much value and emphasis on facial esthetics. Despite evidence showing detrimental effects of dental-facial impairment on social judgments, however, this particular aspect of facial esthetics may be too insignificant to affect self-esteem. Graber and Lucker (1980) suggest that a positive bias exists in self-evaluations of overall satisfaction with dentalfacial esthetics, while evaluations of actual tooth appearance may be more objective. Perhaps adolescents are able to evaluate their dental-facial appearance objectively as negative, without incorporating these judgments into their overall satisfaction with social- and self-worth.

It is possible, of course, that children who make the decision to enter treatment benefit from greater parental support or from more positive selfassessments in ways that support their treatment participation. If such preexisting conditions or attributes are present in the treatment-seeking population, this could minimize expected improvements in self-concept and related variables. Such an explanation would be weakened, however, by the fact that adolescents in this study included both active seekers of treatment at the University Orthodontics Clinic and more passive seekers, who were simply referred by school health personnel to the County Dental Clinic. Since patients were randomly assigned to treatment and control groups, moreover, these influences would have been neutralized.

The results of this study suggest that the role of attractiveness in the formation of self-esteem may have to be reevaluated and reframed. Perhaps adolescent self-esteem is more related to interpersonal performance than to dental-facial esthetics or, even, general physical attractiveness. Given this framework for understanding adolescent self-concept, demonstrable treatment effects would depend on treatment-related changes in self-protective strategies and social interaction outcomes.

In addition, these results call into question the common rationale for providing orthodontic treatment, at least for individuals with mild to moderate malocclusion. Moreover, these findings can be interpreted as emphasizing the overriding importance of maturational processes in the development of self-concept. The full context of adolescent social development, therefore, needs to be considered in decisions related to orthodontic treatment for young people. 
A large body of research, in fact, demonstrates that facial attractiveness and aesthetics play a central role across developmental stages for personal and social life (Albino et al., 1990). Perceptions of self and others clearly help to shape the foundations of self-cognition (viz., attributions, expectations, self-efficacy), but additional research will be needed to determine whether attributions for facial characteristics have constancy before, during, and beyond adolescence. Future studies also should be aimed at identifying relationships between facial appearance conditions and the influence of treatment on cognitions and behaviors, as well as understanding the persistence of these effects.

\section{ACKNOWLEDGMENTS}

The authors gratefully acknowledge the advice, consultation, and clinical services of Drs. John Cunat, Larry Green, and Eugene Lewis during the course of this study. The authors also would like to thank Ms. Mary C. Murphy for her excellent assistance in data collection and management and Drs. Elaine Davis, G. Donald Bissel, and Malcolm Slakter for their many contributions during the design and implementation of this study.

\section{REFERENCES}

Albino, J. E. (1981). Development of methodologies for behavioral measurements related to malocclusion. (Final Report: Contract No. NO1-DE-27499), National Institute of Dental Research, Bethesda, MD.

Albino, J. E. (1990). Effects of orthodontics on psychosocial functioning (Final Report: Contract No. RO1-DE-06154), National Institute of Dental Rescarch, Bethesda, MD.

Albino, J. E., and Tedesco, L. A. (1988). The role of perception in treatment of impaired facial appearance. In Alley, T. R. (ed.), Social and Applied Aspects of Perceiving Faces, Lawrence Erlbaum, Hillsdale, $\mathrm{NJ}$.

Albino, J. E., Lewis, E. A., Wu, T. H., Slakter, M. J., and Fox, R. N. (1979). Comparisons of professional and public assessments of malocclusion. Paper presented at the annual meeting of the International Association for Dental Research, New Orleans, LA.

Albino, J. E., Alley, T. R., Tedesco, L. A., Tobiasen, J. A., Kiyak, H. A., and Lawrence, S. D. (1990). Esthetic issues in behavioral dentistry. Ann. Behav. Med. 12(4): 148-155.

Barocas, R., and Daroly, P. (1972). Effects of physical appearance on social responsiveness. Psychol. Rep. 31: 495-500.

Berscheid, E., and Walster, E. (1974). Physical attractiveness. In Berkowitz, L. (ed.), Advances in Experimental Social Psychology, Vol. 7, Academic Press, New York.

Berscheid, E., Dion, K. K., Walster, E., and Walster, G. W. (1971). Physical attractiveness and dating choice: A test of the matching hypothesis. J. Exp. Soc. Psychol. 7: 173-189.

Cohen, L. K., and Horowitz, H. S. (1970). Occlusal relations in children born and reared in an optimally fluoridated community. III. Social-psychological findings. Angle Orthodont. 40: $159-169$.

Coleman, J. C. (1980). Friendship and the peer group in adolescence. In Adelson, J. (ed.), Handbook of Adolescent Psychology, John Wiley \& Sons, New York, pp. 408-431. 
Coopersmith, S. (1984). Self-Esteem Inventories, Consulting Psychologists Press, Palo Alto, CA. Crandall, V. C., Crandall, V .J., and Katkovsky, W. (1965). A children's social desirability questionnaire. J. Consult. Psychol. 29: 27-36.

Dennington, R. J., and Korabik, K. (1977). Self-concept changes in orthodontic patients during initial treatment. Am. J. Orthodont. 72: 461.

Dion, K. K., Berscheid, E., and Walster, E. (1972). What is beautiful is good. J. Personal. and Soc. Psychol. 24: 285-290.

Fisk, R. O. (1963). Physiological and socio-psychological significance of malocclusion. J. Can. Dent. Assoc. 29: 635-643.

Ford, M. E. (1982). Social cognition and social competence in adolescence. Dev. Psychol. 18: 323-340.

Gochman, D. S. (1972). Some correlates of children's health beliefs and potential health behavior. J. Health Soc. Behav. 12: 148-154.

Gochman, D. S. (1975). The measurement and development of dentally relevant motives. $J$. Public Health Dentist. 35: 160-164.

Goldman, W., and Lewis, P. (1977). Beautiful is good: Evidence that the physically attractive are more socially skillful. J. Exp. Soc. Psychol. 13: 125-130.

Graber, L. W., and Lucker, G. W. (1980). Dental esthetic self-evaluation and satisfaction. Am. J. Orthodont. 77: 163-173.

Grainger, R. M. (1967). Orthodontic Treatment Priority Index, Public Health Service Publ. No. 1000, Series 2, No. 25, U.S. Government Printing Office, Washington, DC.

Jenny, J., Cons, N. C., Kohout, F., and Frazier, P. J. (1980). Test of a method to determine socially acceptable occlusal conditions. J. Dent. Res. 59(A): 11.

Kelly, J. E., and Harvey, C. R. (1977). An assessment of the occlusion of the teeth of youths 12-17 years, United States, Data from the National Health Survey, Series 11, No. 162. DHEW Publ. No. (HRA) 77-1644, Health Resources Administration, National Center for Health Statistics, Rockville, MD.

King, D. L. (1983). Etiology of malocclusion. Birth Defects Orig. Art. Ser. 19: 83-94.

Kiyak, H. A., West, R. A., Hohl, T., and McNeill, R. W. (1982). The psychological impact of orthognathic surgery: A 9-month follow-up. Am. J. Orthodont. 81: 404-412.

Kiyak, H. A., Hohl, T., West, R. A., and McNeill, R. W. (1984). Psychologic changes in orthognathic surgery patients: A 24-month follow-up. J. Oral Maxillofac. Surg. 42: 506-512.

Klima, R. J., Wittemann, J. K., and McIver, J. E. (1979). Body-image, self-concept, and the orthodontic patient. Am. J. Orthodont. 75: 507-516.

Lewis, E. A., Fox, R. N., Albino, J. E., Slakter, M. J., and Wu, T. H. (1979). Accuracy of self-perception of occlusal states. Paper presented at the annual meeting of the International Association for Dental Research, New Orleans, LA.

Lucker, G. W., Graber, L. W., and Pietromonaco, P. (1981). The importance of dentofacial appearance in facial esthetics: A signal detection approach. Basic Appl. Soc. Psychol. 2: 261-274.

Macgregor, F. C. (1970). Social and psychological implications of dentofacial disfigurement. Angle Orthodont. 40: 231-233.

McLain, J. B., and Proffitt, W. R. (1985). Oral health status in the United States: Prevalence of malocclusion. J. Dent. Educ. 49; 386-397.

Pitt, E. J. (1977). Relationship between psychological self-concept and self-perception of the physical profile. Am. J. Orthodont. 72: 459.

Prahi-Anderson, B. (1978). The need for orthodontic treatment. Angle Orthodont. 48: 1-9.

Rosenberg, M. (1974). Malocclusion and craniofacial malformation: Self-concept implications. Paper presented at the Workshop on Psychological Aspects of Craniofacial Malformation, Hilton Head, SC.

Rutzen, S. R. (1973). The social importance of orthodontic rehabilitation: Report of a five year follow-up study. J. Health Soc. Behav. 14: 233-240.

Secord, P. F., and Backman, C. W. (1959). Malocclusion and psychological factors. J. Am. Dent. Assoc. 59: 931-938.

Secord, P. F., and Jourard, S. M. (1953). The appraisal of body cathexis: Body cathexis and the self. J. Consult. Psychol. 17: 343-347. 
Shaw, W. C. (1981). The influence of children's dentofacial appearances on their social attractiveness as judged by peers and lay adults. Am. J. Orthodont. 79: 399-415.

Shaw, W. C., Addy, J., and Ray, C. (1980a). Dental and social effects of malocclusion and effectiveness of orthodontic treatment: A review. Commun. Dentist. Oral Epidemiol. 8: 36-45.

Shaw, W. C., Meek, S. C., and Jones, D. S. (1980b). Nicknames, teasing, harassment and the salience of dental features among school children. Br. J. Orthodont. 7: 75-80.

Simmons, R. G., Rosenberg, R., and Rosenberg, M. (1973). Disturbance in the self-image at adolescence. Am. Sociol. Rev. 38: 553-568.

Slakter, M. J., Albino, J. E., Green, L. J., and Lewis, E. A. (1980). Validity of an orthodontic treatment priority index to measure need for treatment. Am. J. Orthodont. 78: 421-425.

Stricker, G. (1970). Psychological issues pertaining to malocclusion. Am. J. Orthodont. 58: 276-283.

Stricker, G., Clifford, E., Cohen, L. K., Giddon, D. B., Meskin, L. H., and Evans, C. A. (1979). Psychosocial aspects of craniofacial disfigurement: A "state of the art" assessment conducted by the Craniofacial Anomalies Program Branch, the National Institute of Dental Research. Am. J. Orthodont. 76: 410-422.

Tedesco, L. A., Albino, J. E., Cunat, J. J., Green, L. J., Lewis, E. A., and Slakter, M. J. (1983a). A dental-facial attractiveness scale. I. Reliability and validity. Am. J. Orthodont. 83: $38-43$.

Tedesco, L. A., Albino, J. E., Cunat, J. J., Slakter, M. J., and Waltz, K. J. (1983b). A dental-facial attractiveness scale. II. Consistency of perception. Am. J. Orthodont. 83: 44-46. 